\title{
EQUITABLE EDGE COLORING ON TENSOR PRODUCT OF
} GRAPHS

\author{
J. VENINSTINE VIVIK ${ }^{1}$, M.M. AKBAR ALI ${ }^{2}$, and G. GIRIJA ${ }^{3}$ \\ ${ }^{1}$ Department of Mathematics, Karunya Institute of Technology and Sciences, Coimbatore 641 \\ 114, Tamil Nadu, INDIA \\ ${ }^{2}$ Department of Mathematics, Government Arts and Science College, Mettupalyam - 641301 , \\ Tamil Nadu, INDIA. \\ ${ }^{3}$ Department of Mathematics, Government Arts College, Coimbatore - 641 018,Tamil Nadu, \\ INDIA.
}

\begin{abstract}
A graph $G$ is edge colored if different colors are assigned to its edges or lines, in the order of neighboring edges are allotted with least diverse $k$-colors. If each of $k$-colors can be partitioned into color sets and differs by utmost one, then it is equitable. The minimum of $k$-colors required is known as equitably edge chromatic number and symbolized by $\chi_{=}^{\prime}(G)$. Further the impression of equitable edge coloring was first initiated by Hilton and de Werra in 1994. In this paper, we ascertain the equitable edge chromatic number of $P_{m} \otimes P_{n}, P_{m} \otimes C_{n}$ and $K_{1, m} \otimes K_{1, n}$.
\end{abstract}

\section{INTRODUCTION}

In the midst of various coloring concepts of graphs, the motive of equitability in edge coloring on tensor product of graphs is an inventive approach. Graphs considered in this paper are of simple finite sets $V$ and $E$. Each element of $V$ is called its vertices and the elements of $E$ are called its edges, which are the unordered pair of vertices. Therefore $G(V, E)$ is a graph. We use the standard notation of graph theory 1, 2. The minimum number of colors needed to color edges of a graph $G$ is utmost its maximum degree. Since all edges incident to the same vertex must be alloted with distinct colors. Noticeably $\chi^{\prime}(G) \geq \Delta(G)$. In 1964, Vizing 3 conjectured that for every simple graph $\chi^{\prime}(G) \leq \Delta(G)+1$. In 1973, Meyer [4] presented the concept of equitable vertex coloring and its equitable

2020 Mathematics Subject Classification. 05C15, 05C76, 05C38.

Keywords and phrases. Equitable edge coloring, tensor product, path, cycle and star graph. com.in

凶vivikjose@gmail.com-Corresponding author; um_akbar@yahoo.com.in; prof_giri@yahoo.

(D) 0000-0003-3192-003X; 0000-0002-7077-4015; 0000-0003-0812-8378.

(C)2020 Ankara University

Communications Faculty of Sciences University of Ankara-Series A1 Mathematics and Statistics 
chromatic number, which opened the way for introducing equitability in the fields of edge and total coloring.

The concept of equitable edge coloring was defined by Hilton and de Werra 5 and the tensor product of graph was defined by P.M.Weichsel 6 . We have merged both these conception and resolute the equitable edge chromatic number of $P_{m} \otimes P_{n}$, $P_{m} \otimes C_{n}$ and $K_{1, m} \otimes K_{1, n}$. The combined component of each of these graphs enlarges as a new structured graph and has wider applications in the areas of networks, scheduling and assignment domains.

\section{Preliminaries}

Definition 2.1. An edge coloring of a graph $G$ is a function $f: E(G) \longrightarrow C$, where $C$ is a set of distinct colors. For any positive integer $k$, a k-edge coloring is an edge coloring that uses exactly $k$ different colors. A proper edge coloring of a graph is an edge coloring such that no two adjacent edges are assigned the same color. Thus a proper edge coloring $f$ of $G$ is a function $f: E(G) \longrightarrow C$ such that $f(e) \neq f\left(e^{\prime}\right)$ whenever edges $e$ and $e^{\prime}$ are adjacent in $G$.

Definition 2.2. The chromatic index of a graph $G$, denoted $\chi^{\prime}(G)$, is the minimum number of different colors required for a proper edge coloring of $G$. The graph $G$ is $k$-edge-chromatic if $\chi^{\prime}(G)=k$.

Definition 2.3. For $k$-proper edge coloring $f$ of graph $G$, if $\| E_{i}|-| E_{j}|| \leq 1$, $i, j=0,1,2, \ldots, k-1$, where $E_{i}(G)$ is the set of edges of color $i$ in $G$, then $f$ is called a $k$-equitable edge coloring of graph $G$, and

$$
\chi_{=}^{\prime}(G)=\min \{k \text { : there exists a } k \text {-equitable edge-coloring of } G\}
$$

is called the equitable edge chromatic number of graph $G$.

Definition 2.4. [6] The tensor product of $G$ and $H$ is the graph, denoted as $G \otimes H$, whose vertex set is $V(G) \otimes V(H)=V(G \otimes H)$, and for each vertices $(g, h)$ and $\left(g^{\prime}, h^{\prime}\right)$ are adjacent precisely if $g g^{\prime} \in E(G)$ and $h h^{\prime} \in E(H)$. Thus

$$
\begin{aligned}
V(G \otimes H) & =\{(g, h) / g \in V(G) \text { and } h \in V(H)\} \\
E(G \otimes H) & =\left\{(g, h)\left(g^{\prime}, h^{\prime}\right) / g g^{\prime} \in E(G) \text { and } h h^{\prime} \in E(H)\right\} .
\end{aligned}
$$

Lemma 2.5. [1] For any simple graph $G(V, E), \chi_{=}^{\prime} \geq \Delta(G)$.

Lemma 2.6. [1] For any simple graph $G$ and $H, \chi_{=}^{\prime}(G)=\chi^{\prime}(G)$, and if $H \subseteq G$, then $\chi^{\prime}(H) \leq \chi^{\prime}(G)$, where $\chi_{=}^{\prime}(G)$ is the proper edge chromatic number of $G$. So Lemma 2.7 and Lemma 2.8 are obtained.

Lemma 2.7. For any simple graph $G$ and $H$, if $H$ is a subgraph of $G$, then $\chi_{=}^{\prime}(H) \leq \chi_{=}^{\prime}(G)$

Lemma 2.8. For any complete graph $K_{p}$ with order $p$,

$$
\chi_{=}^{\prime}\left(K_{p}\right)= \begin{cases}p, & p \equiv 1(\bmod 2), \\ p-1, & p \equiv 0(\bmod 2),\end{cases}
$$


Lemma 2.9. [7] Let $G(V, E)$ be a connected graph. If there are two adjacent vertices with maximum degree, then $\chi_{a s}^{\prime}(G) \geq \Delta(G)+1$.

\section{Main Results}

Theorem 3.1. For $m \leq n, \chi_{=}^{\prime}\left(P_{m} \otimes P_{n}\right)=4$.

Proof. Let $V\left(P_{m}\right)=\left\{u_{i}: 1 \leq i \leq m\right\}$ and $V\left(P_{n}\right)=\left\{v_{j}: 1 \leq j \leq n\right\}$. By the definiton of tensor product, $V\left(P_{m} \otimes P_{n}\right)=\left\{u_{i} v_{j}: 1 \leq i \leq m, 1 \leq j \leq n\right\}$ and

$E\left(P_{m} \otimes P_{n}\right)=\bigcup_{i=1}^{m-1}\left\{u_{i} v_{j}, u_{i+1} v_{j+1}: 1 \leq j \leq n-1\right\} \cup \bigcup_{i=1}^{m-1}\left\{u_{i} v_{j}, u_{i+1} v_{j-1}: 2 \leq j \leq n\right\}$.

Let $e_{(i)(j),(k)(l)}$ be the edge of $P_{m} \otimes P_{n}$ connecting the vertices $u_{i} v_{j}$ and $u_{k} v_{l}$ of $P_{m} \otimes P_{n}$.

Therefore $e_{(i)(j),(k)(l)} \in E\left(P_{m} \otimes P_{n}\right)$ if and only if $|k-i|=|l-i|=1$. Since $P_{m} \otimes P_{n}$ is isomorphic to $P_{n} \otimes P_{m}$. Without loss of generality, we assume $m \leq n$ for all cases of $m$ and $n$. Now let us partition $E\left(P_{m} \otimes P_{n}\right)$ for the following cases.

Case (i) Both $m$ and $n$ are odd

$$
\begin{aligned}
& E_{1}=\left\{\bigcup_{i=1}^{\frac{m-1}{2}}\left(\bigcup_{j=1}^{\frac{n-1}{2}} e_{(2 i)(2 j-1),(2 i-1)(2 j)}\right)\right\} \bigcup\left\{\bigcup_{i=1}^{\frac{m-1}{2}}\left(\bigcup_{j=1}^{\frac{n-1}{2}} e_{(2 i)(2 j),(2 i+1)(2 j+1)}\right)\right\} \\
& E_{2}=\left\{\bigcup_{i=1}^{\frac{m-1}{2}}\left(\bigcup_{j=1}^{\frac{n-1}{2}} e_{(2 i-1)(2 j-1),(2 i)(2 j)}\right)\right\} \bigcup\left\{\bigcup_{i=1}^{\frac{m-1}{2}}\left(\bigcup_{j=1}^{\frac{n-1}{2}} e_{(2 i+1)(2 j),(2 i)(2 j+1)}\right)\right\} \\
& E_{3}=\left\{\bigcup_{i=1}^{\frac{m-1}{2}}\left(\bigcup_{j=1}^{\frac{n-1}{2}} e_{(2 i+1)(2 j-1),(2 i)(2 j)}\right)\right\} \bigcup\left\{\bigcup_{i=1}^{\frac{m-1}{2}}\left(\bigcup_{j=1}^{\frac{n-1}{2}} e_{(2 i-1)(2 j),(2 i)(2 j+1)}\right)\right\} \\
& E_{4}=\left\{\bigcup_{i=1}^{\frac{m-1}{2}}\left(\bigcup_{j=1}^{\frac{n-1}{2}} e_{(2 i)(2 j-1),(2 i+1)(2 j)}\right)\right\} \bigcup\left\{\bigcup_{i=1}^{\frac{m-1}{2}}\left(\bigcup_{j=1}^{\frac{n-1}{2}} e_{(2 i)(2 j),(2 i-1)(2 j+1)}\right)\right\}
\end{aligned}
$$

In this partition $\left|E_{1}\right|=\left|E_{2}\right|=\left|E_{3}\right|=\left|E_{4}\right|=2\left(\frac{m-1}{2}\right)\left(\frac{n-1}{2}\right)$ and satisfies ||$E_{i}|-| E_{j}|| \leq 1$ for $i \neq j$.

Case (ii) When $m$ is odd and $n$ is even

$$
\begin{aligned}
& E_{1}=\left\{\bigcup_{i=1}^{\frac{m-1}{2}}\left(\bigcup_{j=1}^{\frac{n}{2}} e_{(2 i)(2 j-1),(2 i-1)(2 j)}\right)\right\} \bigcup\left\{\bigcup_{i=1}^{\frac{m-1}{2}}\left(\bigcup_{j=1}^{\frac{n-2}{2}} e_{(2 i)(2 j),(2 i+1)(2 j+1)}\right)\right\} \\
& E_{2}=\left\{\bigcup_{i=1}^{\frac{m-1}{2}}\left(\bigcup_{j=1}^{\frac{n}{2}} e_{(2 i-1)(2 j-1),(2 i)(2 j)}\right)\right\} \bigcup\left\{\bigcup_{i=1}^{\frac{m-1}{2}}\left(\bigcup_{j=1}^{\frac{n-2}{2}} e_{(2 i+1)(2 j),(2 i)(2 j+1)}\right)\right\}
\end{aligned}
$$




$$
\begin{aligned}
& E_{3}=\left\{\bigcup_{i=1}^{\frac{m-1}{2}}\left(\bigcup_{j=1}^{\frac{n}{2}} e_{(2 i+1)(2 j-1),(2 i)(2 j)}\right)\right\}\left\{\bigcup_{i=1}^{\frac{m-1}{2}}\left(\bigcup_{j=1}^{\frac{n-2}{2}} e_{(2 i-1)(2 j),(2 i)(2 j+1)}\right)\right\} \\
& E_{4}=\left\{\bigcup_{i=1}^{\frac{m-1}{2}}\left(\bigcup_{j=1}^{\frac{n}{2}} e_{(2 i)(2 j-1),(2 i+1)(2 j)}\right)\right\} \bigcup\left\{\bigcup_{i=1}^{\frac{m-1}{2}}\left(\bigcup_{j=1}^{\frac{n-2}{2}} e_{(2 i)(2 j),(2 i-1)(2 j+1)}\right)\right\}
\end{aligned}
$$

In this partition $\left|E_{1}\right|=\left|E_{2}\right|=\left|E_{3}\right|=\left|E_{4}\right|=\left(\frac{m-1}{2}\right)\left(\frac{n}{2}\right)+\left(\frac{m-1}{2}\right)\left(\frac{n-2}{2}\right)$

which infers $\| E_{i}|-| E_{j}|| \leq 1$ for $i \neq j$.

Case (iii) When $m$ is even and $n$ is odd

$$
\begin{aligned}
& E_{1}=\left\{\bigcup_{i=1}^{\frac{m}{2}}\left(\bigcup_{j=1}^{\frac{n-1}{2}} e_{(2 i)(2 j-1),(2 i-1)(2 j)}\right)\right\} \bigcup\left\{\bigcup_{i=1}^{\frac{m-2}{2}}\left(\bigcup_{j=1}^{\frac{n-1}{2}} e_{(2 i)(2 j),(2 i+1)(2 j+1)}\right\}\right. \\
& E_{2}=\left\{\bigcup_{i=1}^{\frac{m}{2}}\left(\bigcup_{j=1}^{\frac{n-1}{2}} e_{(2 i-1)(2 j-1),(2 i)(2 j)}\right)\right\} \bigcup\left\{\bigcup_{i=1}^{\frac{m-2}{2}}\left(\bigcup_{j=1}^{\frac{n-1}{2}} e_{(2 i+1)(2 j),(2 i)(2 j+1)}\right)\right\} \\
& E_{3}=\left\{\bigcup_{i=1}^{\frac{m-2}{2}}\left(\bigcup_{j=1}^{\frac{n-1}{2}} e_{(2 i+1)(2 j-1),(2 i)(2 j)}\right)\right\} \bigcup\left\{\bigcup_{i=1}^{\frac{m}{2}}\left(\bigcup_{j=1}^{\frac{n-1}{2}} e_{(2 i-1)(2 j),(2 i)(2 j+1)}\right)\right\} \\
& E_{4}=\left\{\bigcup_{i=1}^{\frac{m-2}{2}}\left(\bigcup_{j=1}^{\frac{n-1}{2}} e_{(2 i)(2 j-1),(2 i+1)(2 j)}\right)\right\} \bigcup\left\{\bigcup_{i=1}^{\frac{m}{2}}\left(\bigcup_{j=1}^{\frac{n-1}{2}} e_{(2 i)(2 j),(2 i-1)(2 j+1)}\right)\right\}
\end{aligned}
$$

In this partition $\left|E_{1}\right|=\left|E_{2}\right|=\left|E_{3}\right|=\left|E_{4}\right|=\left(\frac{m-2}{2}\right)\left(\frac{n-1}{2}\right)+\left(\frac{m}{2}\right)\left(\frac{n-1}{2}\right)$

which facts that $\| E_{i}|-| E_{j}|| \leq 1$ for $i \neq j$.

Case (iv)Both $m$ and $n$ are even

$$
\begin{aligned}
& E_{1}=\left\{\bigcup _ { i = 1 } ^ { \frac { m } { 2 } } ( \bigcup _ { j = 1 } ^ { \frac { n } { 2 } } e _ { ( 2 i ) ( 2 j - 1 ) , ( 2 i - 1 ) ( 2 j ) } \} \bigcup \left\{\bigcup_{i=1}^{\frac{m-2}{2}}\left(\bigcup_{j=1}^{\frac{n-2}{2}} e_{(2 i)(2 j),(2 i+1)(2 j+1)}\right\}\right.\right. \\
& E_{2}=\left\{\bigcup_{i=1}^{\frac{m}{2}}\left(\bigcup_{j=1}^{\frac{n}{2}} e_{(2 i-1)(2 j-1),(2 i)(2 j)}\right\} \bigcup\left\{\bigcup_{i=1}^{\frac{m-2}{2}}\left(\bigcup_{j=1}^{\frac{n-2}{2}} e_{(2 i+1)(2 j),(2 i)(2 j+1)}\right)\right\}\right. \\
& E_{3}=\left\{\bigcup_{i=1}^{\frac{m-2}{2}}\left(\bigcup_{j=1}^{\frac{n}{2}} e_{(2 i+1)(2 j-1),(2 i)(2 j)}\right)\right\} \bigcup\left\{\bigcup_{i=1}^{\frac{m}{2}}\left(\bigcup_{j=1}^{\frac{n-2}{2}} e_{(2 i-1)(2 j),(2 i)(2 j+1)}\right)\right\} \\
& E_{4}=\left\{\bigcup_{i=1}^{\frac{m-2}{2}}\left(\bigcup_{j=1}^{\frac{n}{2}} e_{(2 i)(2 j-1),(2 i+1)(2 j)}\right)\right\} \bigcup\left\{\bigcup_{i=1}^{\frac{m}{2}}\left(\bigcup_{j=1}^{\frac{n-2}{2}} e_{(2 i)(2 j),(2 i-1)(2 j+1)}\right)\right\}
\end{aligned}
$$


In this partition $\left|E_{1}\right|=\left|E_{2}\right|=\left(\frac{m}{2}\right)\left(\frac{n}{2}\right)+\left(\frac{m-2}{2}\right)\left(\frac{n-2}{2}\right)$

and $\left|E_{3}\right|=\left|E_{4}\right|=\left(\frac{m-2}{2}\right)\left(\frac{n}{2}\right)+\left(\frac{m}{2}\right)\left(\frac{n-2}{2}\right)$ which signifies ||$E_{i}|-| E_{j}|| \leq 1$ for $i \neq j$.

In all the cases by observing the suffixes of the edges of $E_{i}$ and $E_{j}(i \neq j)$, it is inferred that there is no common edges in $E_{i}$ and $E_{j}(i \neq j)$. i.e, $E_{i} \cap E_{j}=\phi$ for $i \neq j$. Clearly $E_{i}$ 's are pair wise mutually disjoint, also $\bigcup_{i=1}^{4} E_{i}=E\left(P_{m} \otimes P_{n}\right)$. Here $P_{m} \otimes P_{n}$ is equitably edge colorable with 4 colors. Hence $\chi_{=}^{\prime}\left(P_{m} \otimes P_{n}\right) \leq 4$. Since $\Delta=4$, we have $\chi_{=}^{\prime}\left(P_{m} \otimes P_{n}\right) \geq \chi^{\prime}\left(P_{m} \otimes P_{n}\right) \geq \Delta=4$. This implies $\chi_{=}^{\prime}\left(P_{m} \otimes P_{n}\right) \geq 4$. Therefore $\chi_{=}^{\prime}\left(P_{m} \otimes P_{n}\right)=4$.

Theorem 3.2. For $m \leq n, \chi_{=}^{\prime}\left(P_{m} \otimes C_{n}\right)=4$.

Proof. Let $V\left(P_{m}\right)=\left\{u_{i}: 1 \leq i \leq m\right\}$ and $V\left(C_{n}\right)=\left\{v_{j}: 1 \leq j \leq n\right\}$. By the definiton of tensor product, $V\left(P_{m} \otimes C_{n}\right)=\left\{u_{i} v_{j}: 1 \leq i \leq m, 1 \leq j \leq n\right\}$ and $E\left(P_{m} \otimes C_{n}\right)=\bigcup_{i=1}^{m-1}\left\{u_{i} v_{j}, u_{i+1} v_{j+1}: 1 \leq j \leq n-1\right\} \cup \bigcup_{i=1}^{m-1}\left\{u_{i} v_{j}, u_{i+1} v_{j-1}: 2 \leq j \leq n\right\}$

$\cup \bigcup_{i=1}^{m-1}\left\{u_{i} v_{1}, u_{i+1} v_{n}\right\} \cup \bigcup_{i=2}^{m}\left\{u_{i} v_{1}, u_{i-1} v_{n}\right\}$. Let $e_{(i)(j),(k)(l)}$ be the edge of $P_{m} \otimes C_{n}$ connecting the vertices $u_{i} v_{j}$ and $u_{k} v_{l}$ of $P_{m} \otimes C_{n}$.

Therefore $e_{(i)(j),(k)(l)} \in E\left(P_{m} \otimes C_{n}\right)$ if and only if $|k-i|=|l-i|=1$. Since $P_{m} \otimes C_{n}$ is isomorphic to $C_{n} \otimes P_{m}$. Without loss of generality, we assume $m \leq n$ for all cases of $m$ and $n$. Now let us partition $E\left(P_{m} \otimes C_{n}\right)$ for the following cases.

Case (i) Both $m$ and $n$ are odd

$$
\begin{aligned}
& E_{1}=\left\{\bigcup_{i=1}^{\frac{m-1}{2}}\left(\bigcup_{j=1}^{\frac{n-1}{2}} e_{(2 i)(2 j-1),(2 i-1)(2 j)}\right)\right\} \bigcup\left\{\bigcup_{i=1}^{\frac{m-1}{2}}\left(\bigcup_{j=1}^{\frac{n-1}{2}} e_{(2 i)(2 j),(2 i+1)(2 j+1)}\right)\right\} \\
& E_{2}=\left\{\bigcup_{i=1}^{\frac{m-1}{2}}\left(\bigcup_{j=1}^{\frac{n-1}{2}} e_{(2 i-1)(2 j-1),(2 i)(2 j)}\right)\right\} \bigcup\left\{\bigcup_{i=1}^{\frac{m-1}{2}}\left(\bigcup_{j=1}^{\frac{n-1}{2}} e_{(2 i+1)(2 j),(2 i)(2 j+1)}\right)\right\} \\
& E_{3}=\left\{\bigcup_{i=1}^{\frac{m-1}{2}}\left(\bigcup_{j=1}^{\frac{n-1}{2}} e_{(2 i+1)(2 j-1),(2 i)(2 j)}\right)\right\} \bigcup\left\{\bigcup_{i=1}^{\frac{m-1}{2}}\left(\bigcup_{j=1}^{\frac{n-1}{2}} e_{(2 i-1)(2 j),(2 i)(2 j+1)}\right)\right\} \\
& E_{4}=\left\{\bigcup_{i=1}^{\frac{m-1}{2}}\left(\bigcup_{j=1}^{\frac{n-1}{2}} e_{(2 i)(2 j-1),(2 i+1)(2 j)}\right)\right\} \bigcup\left\{\bigcup_{i=1}^{\frac{m-1}{2}}\left(\bigcup_{j=1}^{\frac{n-1}{2}} e_{(2 i)(2 j),(2 i-1)(2 j+1)}\right)\right\}
\end{aligned}
$$




$$
\bigcup\left\{e_{(2 i-1)(1),(2 i)(n): 1 \leq i \leq \frac{m-1}{2}}\right\}
$$

In this partition $\left|E_{1}\right|=\left|E_{2}\right|=\left|E_{3}\right|=\left|E_{4}\right|=2\left(\frac{m-1}{2}\right)\left(\frac{n-1}{2}\right)+\left(\frac{m-1}{2}\right)$.

and deduce that ||$E_{i}|-| E_{j}|| \leq 1$ for $i \neq j$.

Case (ii) When $m$ is odd and $n$ is even

$$
\begin{aligned}
& E_{1}=\left\{\bigcup_{i=1}^{\frac{m-1}{2}}\left(\bigcup_{j=1}^{\frac{n}{2}} e_{(2 i)(2 j-1),(2 i-1)(2 j)}\right)\right\} \bigcup\left\{\bigcup_{i=1}^{\frac{m-1}{2}}\left(\bigcup_{j=1}^{\frac{n-2}{2}} e_{(2 i)(2 j),(2 i+1)(2 j+1)}\right)\right\} \\
& \bigcup\left\{e_{(2 i+1)(1),(2 i)(n): 1 \leq i \leq \frac{m-1}{2}}\right\} \\
& E_{2}=\left\{\bigcup_{i=1}^{\frac{m-1}{2}}\left(\bigcup_{j=1}^{\frac{n}{2}} e_{(2 i-1)(2 j-1),(2 i)(2 j)}\right)\right\} \bigcup\left\{\bigcup_{i=1}^{\frac{m-1}{2}}\left(\bigcup_{j=1}^{\frac{n-2}{2}} e_{(2 i+1)(2 j),(2 i)(2 j+1)}\right)\right\} \\
& \bigcup\left\{e_{(2 i)(1),(2 i+1)(n): 1 \leq i \leq \frac{m-1}{2}}\right\} \\
& E_{3}=\left\{\bigcup_{i=1}^{\frac{m-1}{2}}\left(\bigcup_{j=1}^{\frac{n}{2}} e_{(2 i+1)(2 j-1),(2 i)(2 j)}\right)\right\} \bigcup\left\{\bigcup_{i=1}^{\frac{m-1}{2}}\left(\bigcup_{j=1}^{\frac{n-2}{2}} e_{(2 i-1)(2 j),(2 i)(2 j+1)}\right)\right\} \\
& \bigcup\left\{e_{(2 i)(1),(2 i-1)(n): 1 \leq i \leq \frac{m-1}{2}}\right\} \\
& E_{4}=\left\{\bigcup_{i=1}^{\frac{m-1}{2}}\left(\bigcup_{j=1}^{\frac{n}{2}} e_{(2 i)(2 j-1),(2 i+1)(2 j)}\right)\right\} \bigcup\left\{\bigcup_{i=1}^{\frac{m-1}{2}}\left(\bigcup_{j=1}^{\frac{n-2}{2}} e_{(2 i)(2 j),(2 i-1)(2 j+1)}\right)\right\} \\
& \bigcup\left\{e_{(2 i-1)(1),(2 i)(n): 1 \leq i \leq \frac{m-1}{2}}\right\}
\end{aligned}
$$

In this partition $\left|E_{1}\right|=\left|E_{2}\right|=\left|E_{3}\right|=\left|E_{4}\right|=\left(\frac{m-1}{2}\right)\left(\frac{n}{2}\right)+\left(\frac{m-1}{2}\right)\left(\frac{n-2}{2}\right)+\left(\frac{m-1}{2}\right)$. and assures that ||$E_{i}|-| E_{j}|| \leq 1$ for $i \neq j$.

Case (iii) When $m$ is even and $n$ is odd

$$
\begin{aligned}
E_{1}= & \left\{\bigcup_{i=1}^{\frac{m}{2}}\left(\bigcup_{j=1}^{\frac{n-1}{2}} e_{(2 i)(2 j-1),(2 i-1)(2 j)}\right)\right\}\left\{\bigcup_{i=1}^{\frac{m-2}{2}}\left(\bigcup_{j=1}^{\frac{n-1}{2}} e_{(2 i)(2 j),(2 i+1)(2 j+1)}\right)\right\} \\
& \bigcup\left\{e_{\left.(2 i+1)(1),(2 i)(n): 1 \leq i \leq \frac{m-2}{2}\right\}}\right. \\
E_{2}= & \left\{\bigcup_{i=1}^{\frac{m}{2}}\left(\bigcup_{j=1}^{\frac{n-1}{2}} e_{(2 i-1)(2 j-1),(2 i)(2 j)}\right)\right\} \bigcup\left\{\bigcup_{i=1}^{\frac{m-2}{2}}\left(\bigcup_{j=1}^{\frac{n-1}{2}} e_{(2 i+1)(2 j),(2 i)(2 j+1)}\right)\right\} \\
& \bigcup\left\{e_{(2 i)(1),(2 i+1)(n): 1 \leq i \leq \frac{m-2}{2}}\right\}
\end{aligned}
$$




$$
\begin{aligned}
E_{3}= & \left\{\bigcup_{i=1}^{\frac{m-2}{2}}\left(\bigcup_{j=1}^{\frac{n-1}{2}} e_{(2 i+1)(2 j-1),(2 i)(2 j)}\right)\right\}\left\{\bigcup_{i=1}^{\frac{m}{2}}\left(\bigcup_{j=1}^{\frac{n-1}{2}} e_{(2 i-1)(2 j),(2 i)(2 j+1)}\right\}\right. \\
& \bigcup\left\{e_{(2 i)(1),(2 i-1)(n): 1 \leq i \leq \frac{m}{2}}\right\} \\
E_{4}= & \left\{\bigcup_{i=1}^{\frac{m-2}{2}}\left(\bigcup_{j=1}^{\frac{n-1}{2}} e_{(2 i)(2 j-1),(2 i+1)(2 j)}\right)\right\} \bigcup\left\{\bigcup_{i=1}^{\frac{m}{2}}\left(\bigcup_{j=1}^{\frac{n-1}{2}} e_{(2 i)(2 j),(2 i-1)(2 j+1)}\right)\right\} \\
& \bigcup\left\{e_{(2 i-1)(1),(2 i)(n): 1 \leq i \leq \frac{m}{2}}\right\}
\end{aligned}
$$

In this partition $\left|E_{1}\right|=\left|E_{2}\right|=\left(\frac{m}{2}\right)\left(\frac{n-1}{2}\right)+\left(\frac{m-2}{2}\right)\left(\frac{n-1}{2}\right)+\left(\frac{m-2}{2}\right)$,

$\left|E_{3}\right|=\left|E_{4}\right|=\left(\frac{m-2}{2}\right)\left(\frac{n-1}{2}\right)+\left(\frac{m}{2}\right)\left(\frac{n-1}{2}\right)+\left(\frac{m}{2}\right)$ and verifies that ||$E_{i}|-| E_{j}|| \leq 1$ for $i \neq j$.

Case (iv) Both $m$ and $n$ are even

$$
\begin{aligned}
E_{1}= & \left\{\bigcup_{i=1}^{\frac{m}{2}}\left(\bigcup_{j=1}^{\frac{n}{2}} e_{(2 i)(2 j-1),(2 i-1)(2 j)}\right)\right\} \bigcup\left\{\bigcup_{i=1}^{\frac{m-2}{2}}\left(\bigcup_{j=1}^{\frac{n-2}{2}} e_{(2 i)(2 j),(2 i+1)(2 j+1)}\right)\right\} \\
& \bigcup\left\{e_{\left.(2 i+1)(1),(2 i)(n): 1 \leq i \leq \frac{m-2}{2}\right\}}\right. \\
E_{2}= & \left\{\bigcup_{i=1}^{\frac{m}{2}}\left(\bigcup_{j=1}^{\frac{n}{2}} e_{(2 i-1)(2 j-1),(2 i)(2 j)}\right)\right\}\left\{\bigcup_{i=1}^{\frac{m-2}{2}}\left(\bigcup_{j=1}^{\frac{n-2}{2}} e_{(2 i+1)(2 j),(2 i)(2 j+1)}\right)\right\} \\
& \bigcup\left\{e_{\left.(2 i)(1),(2 i+1)(n): 1 \leq i \leq \frac{m-2}{2}\right\}}\right. \\
E_{3}= & \left\{\bigcup_{i=1}^{\frac{m-2}{2}}\left(\bigcup_{j=1}^{\frac{n}{2}} e_{(2 i+1)(2 j-1),(2 i)(2 j)}\right)\right\} \bigcup\left\{\bigcup_{i=1}^{\frac{m}{2}}\left(\bigcup_{j=1}^{\frac{n-2}{2}} e_{(2 i-1)(2 j),(2 i)(2 j+1)}\right)\right\} \\
& \bigcup\left\{e_{\left.(2 i)(1),(2 i-1)(n): 1 \leq i \leq \frac{m}{2}\right\}}\right. \\
E_{4}= & \left\{\bigcup_{i=1}^{\frac{m-2}{2}}\left(\bigcup_{j=1}^{\frac{n}{2}} e_{(2 i)(2 j-1),(2 i+1)(2 j)}\right)\right\} \bigcup\left\{\bigcup_{i=1}^{\frac{m}{2}}\left(\bigcup_{j=1}^{\frac{n-2}{2}} e_{(2 i)(2 j),(2 i-1)(2 j+1)}\right)\right\} \\
& \bigcup\left\{e_{\left.(2 i-1)(1),(2 i)(n): 1 \leq i \leq \frac{m}{2}\right\}}\right.
\end{aligned}
$$

In this partition $\left|E_{1}\right|=\left|E_{2}\right|=\left(\frac{m}{2}\right)\left(\frac{n}{2}\right)+\left(\frac{m-2}{2}\right)\left(\frac{n-2}{2}\right)+\left(\frac{m-2}{2}\right)$ and $\left|E_{3}\right|=\left|E_{4}\right|=\left(\frac{m-2}{2}\right)\left(\frac{n}{2}\right)+\left(\frac{m}{2}\right)\left(\frac{n-2}{2}\right)+\left(\frac{m}{2}\right)$ which is evident that ||$E_{i}|-| E_{j}|| \leq 1$ for $i \neq j$.

In all the cases by observing the suffixes of the edges of $E_{i}$ and $E_{j}(i \neq j)$, it is inferred that there is no common edges in $E_{i}$ and $E_{j}(i \neq j)$ and implies 
$E_{i} \cap E_{j}=\phi$ for $i \neq j$. Clearly $E_{i}^{\prime} s$ are pair wise mutually disjoint, also $\bigcup_{i=1}^{4} E_{i}$ $=E\left(P_{m} \otimes C_{n}\right)$. Here $P_{m} \otimes C_{n}$ is equitably edge colorable with 4 colors. Hence $\chi_{=}^{\prime}\left(P_{m} \otimes C_{n}\right) \leq 4$. Since $\Delta=4, \chi_{=}^{\prime}\left(P_{m} \otimes C_{n}\right) \geq \chi^{\prime}\left(P_{m} \otimes C_{n}\right) \geq \Delta=4$. This implies $\chi_{=}^{\prime}\left(P_{m} \otimes C_{n}\right) \geq 4$. Therefore $\chi_{=}^{\prime}\left(P_{m} \otimes C_{n}\right)=4$.

Theorem 3.3. For any positive integer $m$ and $n, \chi_{=}^{\prime}\left(K_{1, m} \otimes K_{1, n}\right)=m n$.

Proof. Let $V\left(K_{1, m}\right)=\left\{u_{0}\right\} \bigcup\left\{u_{i}: 1 \leq i \leq m\right\}$ and $V\left(K_{1, n}\right)=\left\{v_{0}\right\}\left\{v_{j}: 1 \leq j \leq n\right\}$

By the definition of tensor product,

$V\left(K_{1, m} \otimes K_{1, n}\right)=\left\{u_{i} v_{j}: 0 \leq i \leq m, 0 \leq j \leq n\right\}$ and

$E\left(K_{1, m} \otimes K_{1, n}\right)=\left\{\bigcup_{i=1}^{m}\left(u_{0} v_{0}, u_{i} v_{j}\right): 1 \leq j \leq n\right\} \bigcup\left\{\bigcup_{i=1}^{m}\left\{\left(u_{i} v_{0}, u_{0} v_{j}\right): 1 \leq j \leq n\right\}\right.$

Let $e_{(i)(j),(k)(l)}$ be the edge of $K_{1, m} \otimes K_{1, n}$ connecting the vertices $u_{i} v_{j}$ and $u_{k} v_{l}$ of the tensor product of star graphs. Since $K_{1, m} \otimes K_{1, n}$ is isomorphic to $K_{1, n} \otimes K_{1, m}$. Without loss of generality, we assume $m \leq n$ for all cases of $m$ and $n$.

Now we partition the edge set of $E\left(K_{1, m} \otimes K_{1, n}\right)$ as

$E_{k}=\left\{e_{(0)(0),\left(\left\lceil\frac{k}{n}\right\rceil\right)((k-1 \bmod n)+1)}\right\} \bigcup\left\{e_{\left(\left\lceil\frac{k}{n}\right\rceil\right)(0),(0)((k-1 \bmod n)+1)}\right\}, 1 \leq k \leq m n$

Clearly each of edge classes $E_{1}, E_{2}, \ldots E_{k}$ are independent sets of $E\left(K_{1, m} \otimes K_{1, n}\right)$, such that $\left|E_{1}\right|=\left|E_{k}\right|=2$. It satisfies the inequality ||$E_{i}|-| E_{j}|| \leq 1$ for every pair $(i, j)$. This implies

$$
\chi_{=}^{\prime}\left(K_{1, m} \otimes K_{1, n}\right) \leq m n . \text { But } \chi_{=}^{\prime}\left(K_{1, m} \otimes K_{1, n}\right) \geq \chi^{\prime}\left(K_{1, m} \otimes K_{1, n}\right) \geq m n .
$$

Therefore $\chi_{=}^{\prime}\left(K_{1, m} \otimes K_{1, n}\right)=m n$.

\section{Conclusion}

The equitable edge coloring of tensor product of graphs is a new inventive approach and this field of research is wide open. The equitable edge coloration of Mycielskian of some graphs are obtained by Vivik and Girija 8]. The proofs provided in this paper establishes an optimal solution for the equitable edge coloring of tensor product of two different paths, paths with cycles and two star graphs. It would be further interesting to discern the bounds of equitable edge coloring of tensor and other product of graphs.

\section{REFERENCES}

[1] Bondy, J. A., Murty, U. S. R., Graph Theory with Applications, New York; The Macmillan Press Ltd., 1976.

[2] Harary, F., Graph Theory, Narosa Publishing home, 1969.

[3] Vizing, V.G., On an estimate of the chromatic class of a p-graph, Metody Diskret. Analiz, 5 (1964), 25-30.

[4] Meyer, W., Equitable Coloring, Amer. Math. Monthly, 80 (1973), 920-922.

[5] Hilton, A.J.W., de Werra, D., A sufficient condition for equitable edge-colorings of simple graphs, Discrete Mathematics, 128 (1994), 179-201.

[6] Weichsel, P.M., The Kronecker product of graphs, Proc. Amer. Math. Soc., 8 (1962), 47-52. 
[7] Li, J.W., Zhang, Z.F., Chen, X.E., Sun, Y.R., A note on adjacent strong edge coloring of $K(n, m)$, Acta Mathematicae Application Sinica, 22(2) (2006), 273-276.

[8] Veninstine Vivik.J and Girija.G, Equitable edge chromatic number of mycielskian of graphs, Far East Journal of Mathematics,101(9) (2017), 1887-1895. 